\title{
Correction: COVID-19 Discourse on Twitter in Four Asian Countries: Case Study of Risk Communication
}

Sungkyu Park ${ }^{1 *}, \mathrm{PhD}$; Sungwon Han ${ }^{2 *}, \mathrm{MSc}$; Jeongwook Kim ${ }^{2 *}$, MSc; Mir Majid Molaie ${ }^{2}, \mathrm{BSc}$; Hoang Dieu Vu ${ }^{3}$, MSc; Karandeep Singh ${ }^{1}$, PhD; Jiyoung Han ${ }^{2}, \mathrm{PhD}$; Wonjae Lee ${ }^{2}$, PhD; Meeyoung Cha ${ }^{1,2}, \mathrm{PhD}$

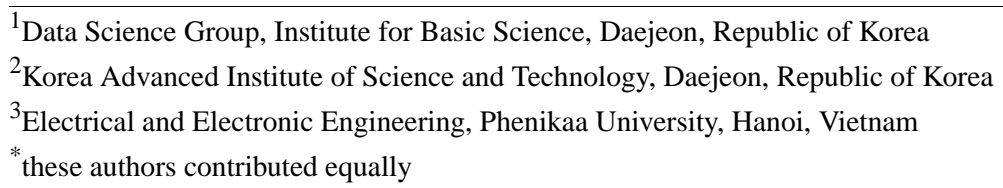

\section{Corresponding Author:}

Meeyoung Cha, $\mathrm{PhD}$

Data Science Group

Institute for Basic Science

55, Expo-ro, Yuseong-gu

Daejeon, 34126

Republic of Korea

Phone: 82428788114

Fax: 82428788079

Email: meeyoung.cha@gmail.com

\section{Related Article:}

Correction of: https://www.jmir.org/2021/3/e23272/

(J Med Internet Res 2021;23(3):e28926) doi: $10.2196 / 28926$

In "COVID-19 Discourse on Twitter in Four Asian Countries: Case Study of Risk Communication" (J Med Internet Res 2021;23(3):e23272) the authors noted one error.

In the originally published article, the caption of Figure 10 was incorrectly described. It read as follows:

Daily topic trends in Vietnam based on the number of tweets.
Daily topic trends in India based on the number of tweets.

The correction will appear in the online version of the paper on the JMIR Publications website on March 29, 2021, together with the publication of this correction notice. Because this was made after submission to PubMed, PubMed Central, and other full-text repositories, the corrected article has also been resubmitted to those repositories.

This has been corrected to:

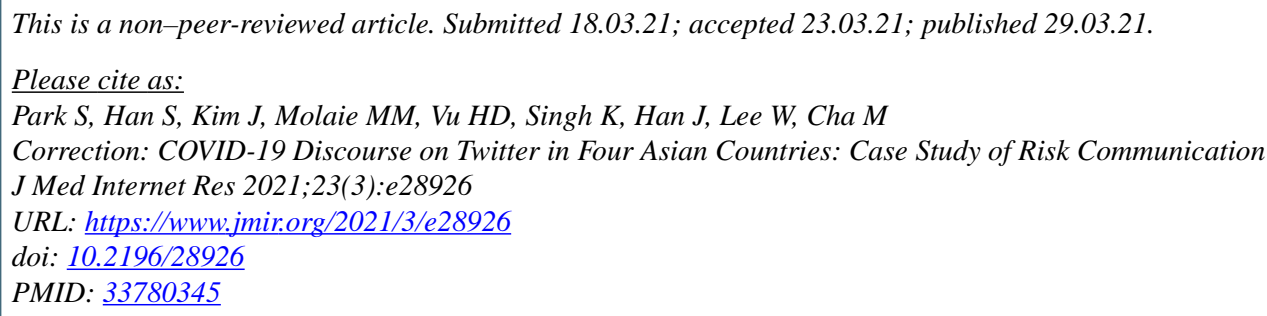

(CSungkyu Park, Sungwon Han, Jeongwook Kim, Mir Majid Molaie, Hoang Dieu Vu, Karandeep Singh, Jiyoung Han, Wonjae Lee, Meeyoung Cha. Originally published in the Journal of Medical Internet Research (http://www.jmir.org), 29.03.2021. This is an open-access article distributed under the terms of the Creative Commons Attribution License (https://creativecommons.org/licenses/by/4.0/), which permits unrestricted use, distribution, and reproduction in any medium, provided the original work, first published in the Journal of Medical Internet Research, is properly cited. The complete bibliographic 
information, a link to the original publication on http://www.jmir.org/, as well as this copyright and license information must be included. 